\title{
Variabilidade espacial de populações de Diatraea saccharalis em canaviais e sugestão de método de amostragem
}

\author{
Leila Luci Dinardo-Miranda (1*); Juliano Vilela Fracasso ('); Dilermando Perecin ( ${ }^{2}$ ) \\ (') Instituto Agronômico, Centro de Cana-de-açúcar, Caixa Postal 206, 14001-970 Ribeirão Preto (SP). \\ (2) Faculdade de Ciências Agrárias e Veterinárias (UNESP/FCAV), Departamento de Ciências Exatas, Via de Acesso Prof. Paulo \\ Donato Castellane, $s / n^{\circ}, 14884-900$ Jaboticabal (SP). \\ (*) Autora correspondente: leiladinardo@iac.sp.gov.br
}

Recebido: 16/ago./2010; Aceito: 12/abr./2011

\begin{abstract}
Resumo
A broca Diatraea saccharalis é uma das mais importantes pragas da cana-de-açúcar no Brasil. O manejo de áreas infestadas está embasado no controle biológico com Cotesia flavipes, cujas liberações são feitas em função da população de lagartas da praga em campo. Por esta razão, a estimativa desta população é de fundamental importância para o sucesso do programa de manejo. Assim, o objetivo do presente trabalho foi estudar a variabilidade espacial de D. saccharalis em canaviais e sugerir um método de amostragem que permitisse uma estimativa adequada das populações em campo. Seis campos de aproximadamente 1 ha cada um foram amostrados, em grid retangular de aproximadamente $10 \times 10 \mathrm{~m}$, com ilhas. Cada ponto de amostragem foi representado pela coleta das formas biológicas da praga no interior dos colmos em $2 \mathrm{~m}$ de sulco. Na maioria das áreas, as populações de formas imaturas de D. saccharalis distribuíram-se de forma contagiosa, com alcance do semivariograma variando de 18 a $80 \mathrm{~m}$. Com base no alcance médio, estimou-se em seis o número médio de pontos de amostragem por hectare que deveriam ser feitos para representar adequadamente a área. Este número foi menor do que o estimado utilizando parâmetros da estatística clássica. Sugere-se que, para estimativa das formas imaturas de $D$. saccharalis em canaviais, a amostragem seja feita em grid de 40 × 40 m, o que resulta em aproximadamente seis pontos de amostragem por hectare e permitirá, na maioria das áreas, efetuar levantamentos com independência e coeficientes de variação menores que $50 \%$.
\end{abstract}

Palavras-chave: broca da cana, cana-de-açúcar, manejo.

\section{Spatial variability of Diatraea saccharalis populations in sugarcane fields and suggestion of sampling method}

\section{Abstract}

The sugarcane borer Diatraea saccharalis Fabr. (Lepidoptera: Crambidae) is one of the most important pests of sugarcane in Brazil. The management of infested area is based on biological control with Cotesia flavipes, whose releases are made according to the pest population in field. Therefore, the evaluation of $D$. saccharalis population is important for the success of management program. The objective of this work was to study the spatial variability of $D$. saccharalis in sugarcane and suggesting a sampling method that allows an adequate evaluation of the population in the field. Six fields with areas of 1 ha were sampled, within a grid measuring about $10 \times 10 \mathrm{~m}$, with islands. Each sample point was represented by collecting biological forms of the pest in the stalks, considering $2 \mathrm{~m}$ of furrow. In most areas, $D$. saccaralis has aggregated spatial distribution and the values of range in semivariogram varied from 18 to $80 \mathrm{~m}$. Based on average range, it was estimated in six the number of samples per hectare to evaluate the population in a given area. This number was smaller than that estimated using classical statistical parameters. According to the results, to obtain a reliable evaluation of the population of $D$. saccharalis immature forms in sugarcane fields, samples should be collected $40 \mathrm{~m}$ apart, meaning about 6 sampling points per hectare. This method allows independent samplings, with coefficients of variation less than $50 \%$ in most areas.

Key words: sugarcane borer, sugarcane, management. 


\section{INTRODUÇÃO}

A broca Diatraea saccharalis Fabr. (Lepidoptera: Crambidae) é uma das mais importantes pragas da cana-de-açúcar no Brasil, e está amplamente distribuída em todas as regiôes canavieiras do país (Dinardo-Miranda, 2008). O dano é causado pelas lagartas que abrem galerias no interior dos colmos, causando morte de grande número de perfilhos, quando o canavial é jovem, e acentuada redução de produtividade, em canaviais mais desenvolvidos, pois os colmos atacados perdem massa, são menores e mais finos, muitos secam e morrem, enquanto outros se quebram pela ação do vento (TerÁn et al., 1985; PreCETti et al., 1988, Botelho e Macedo, 2002).

Em condições favoráveis, pelos orifícios feitos pelas lagartas ainda entram pragas secundárias, como Metamasius hemipterus (L.) (Coleoptera: Curculioniodae), que acentuam as perdas no campo (Precetti et al., 1988). Também é comum a entrada de diversos microrganismos, especialmente fungos, que causam a "podridão vermelha", com reduçấo do teor de açúcar nos colmos devido à inversão da sacarose armazenada na planta e sua transformaçáo em glicose e levulose. $\mathrm{Na}$ indústria, os microrganismos presentes no colmo contaminam o caldo, prejudicando os processos industriais, por dificultar a obtenção de açúcar de qualidade e inibir a fermentação (Botelho e Macedo, 2002; Stupiello, 2005, Dinardo-Miranda, 2008).

Trabalhos realizados pela Copersucar, com algumas variedades em cultivo no fim da década de 1990 e apresentados por ARRIGONI (2002), revelaram que a cada $1 \%$ de intensidade de infestação da broca, as perdas poderiam chegar a $1,50 \%$ na produtividade de colmos, $0,49 \%$ na produtividade de açúcar e $0,28 \%$ na produtividade de álcool, dados que ilustram a importância da praga para a cultura.

Embora $D$. saccharalis e cana-de-açúcar sejam de grande relevância para a economia do Brasil, são raros e antigos os estudos sobre distribuição espacial desses insetos em campo e tais estudos são imprescindíveis para o desenvolvimento de planos de amostragem, visando à aplicaçáo em programas de manejo integrado de pragas (TAYlOR, 1984; Southwood, 1978; Giles et al., 2000).

No Brasil, o manejo da broca está embasado no controle biológico, especialmente envolvendo o parasitóide de lagartas Cotesia flavipes, cujo número de adultos liberados no canavial atacado depende da população de lagartas da broca em campo (Dinardo-Miranda, 2008).

O trabalho pioneiro no Brasil sobre métodos para estimar as populaçóes de formas imaturas de D. saccharalis em canaviais foi realizado por TERÁN (1988), que concluiu que as amostragens deveriam ser feitas em 0,28\% dos sulcos do talhão, o que, nas situaçôes em estudo, representavam avaliar $20 \mathrm{~m}$ de sulcos por ha. O autor ressaltou, entretanto, que deveriam ser amostrados muitos pontos por hectare, de maneira que na totalidade $20 \mathrm{~m} \mathrm{ha}^{-1}$ de sulcos fossem amostrados.

Apesar da recomendação de Terán (1988), muitos produtores adotaram a estratégia de avaliar somente 2 pontos por hectare, sendo cada ponto representado por $10 \mathrm{~m}$ de sulco; há produtores que amostram número ainda menor de pontos por hectare (DinARDo-Miranda, 2008) e outros que fazem os levantamentos coletando colmos com sintoma de ataque de broca, durante caminhamento ao acaso no talhão, por determinado período, como recomendado por MACEDO e BotelHo (1988).

Visto que a liberação de parasitóides é feita em função da população da broca em campo, a estimativa da população da praga é de fundamental importância para o sucesso do programa de manejo. Desta forma, o objetivo do presente trabalho foi estudar a distribuição espacial de D. saccharalis em canaviais e, com base nela, sugerir um método de amostragem que permita uma estimativa adequada das populaçóes em campo.

\section{MATERIAL E MÉTODOS}

A distribuição espacial de lagartas de $D$. saccharalis foi estudada em seis áreas comercias, cujas características estão na tabela 1 .

Em cada campo experimental, selecionou-se área de aproximadamente $1 \mathrm{ha}$, onde se procedeu a amostragem em grade retangular (Área 1), ou em grade retangular com cinco ilhas (Áreas 2 a 6), nas quais as amostras foram coletadas mais próximas umas das outras (Tabela 1).

$\mathrm{O}$ ponto de amostragem foi representado por $2 \mathrm{~m}$ de sulco, no qual se procedeu a retirada da palha de todos os colmos, para permitir a visualização de sintomas de ataque da broca. Colmos com sintoma foram retirados da touceira e abertos longitudinalmente, para identificaçáo e contagem das formas biológicas presentes, ou seja, lagartas sadias, pupas e lagartas parasitadas por $C$. flavipes.

Com os dados obtidos referentes a lagartas e formas biológicas totais, calcularam-se os parâmetros estatísticos básicos (média, desvio-padrão, coeficiente de variação, valores mínimos e máximos, assimetria e curtose). Em seguida, verificou-se a hipótese de normalidade dos dados, pelo teste de Shapiro e WiLK (1965) e calcularamse os índices de Morisita, como descrito em Silveira Neto et al. (1976).

A seguir, procederam-se às análises geoestatísticas dos dados, usando semivariogramas e interpolação por krigagem para construçáo de mapas, como descrito por VIEIRA et al. (1983). As análises de semivariograma foram feitas utilizando o programa GEOSTAT (VIEIRA et al., 1983). Com os modelos ajustados para os semivariogramas, utilizou-se o teste de jackknifing para verificar se as estimativas dos parâmetros dos semivariogramas estavam adequados e para estimar qual o número de vizinhos a serem utilizados 
Tabela 1. Características das áreas amostradas

\begin{tabular}{|c|c|c|c|c|c|c|}
\hline Características & Área 1 & Área 2 & Área 3 & Área 4 & Área 5 & Área 6 \\
\hline Local & Orindiuva & Orindiuva & Sertãozinho & Guaíra & Orindiuva & Orindiuva \\
\hline Variedade & SP81-3250 & RB925211 & RB867515 & SP80-3280 & RB867515 & RB855453 \\
\hline Ciclo & cana planta & cana planta & cana planta & soca de $4 .^{\circ}$ corte & soca de $2 .^{\circ}$ corte & soca de $3 .^{\circ}$ corte \\
\hline Idade (meses) & 8 & 6 & 7 & 3 & 5 & 4 \\
\hline $\begin{array}{l}\text { Data da } \\
\text { amostragem }\end{array}$ & $28 / 11 / 07$ & 12/1/09 & $13 / 11 / 08$ & $23 / 2 / 10$ & $31 / 3 / 10$ & $1 . \% / 4 / 10$ \\
\hline $\begin{array}{l}\text { Grid de } \\
\text { amostragem }\end{array}$ & $9,8 \times 10 \mathrm{~m}$ & $\begin{array}{l}12 \times 12 \mathrm{~m} \\
\text { com } 5 \text { ilhas } \\
\text { de } 6 \times 6 \mathrm{~m}\end{array}$ & $\begin{array}{c}15 \times 10 \mathrm{~m} \\
\text { com } 5 \text { ilhas } \\
\text { de } 7,5 \times 5 \mathrm{~m}\end{array}$ & $\begin{array}{l}10,8 \times 10 \mathrm{~m} \\
\text { com } 5 \text { ilhas } \\
\text { de } 5,4 \times 5 \mathrm{~m}\end{array}$ & $\begin{array}{c}10,5 \times 10 \mathrm{~m} \\
\text { com } 5 \text { ilhas } \\
\text { de } 5,25 \times 5 \mathrm{~m}\end{array}$ & $\begin{array}{c}10,5 \times 10 \mathrm{~m} \\
\text { com ilhas } \\
\text { de } 5,25 \times 5 \mathrm{~m}\end{array}$ \\
\hline N. ${ }^{\circ}$ de amostras & 150 & 150 & 140 & 120 & 120 & 120 \\
\hline $\begin{array}{l}\text { Área amostrada } \\
\text { (ha) }\end{array}$ & 1,23 & 0,86 & 1,28 & 0,98 & 0,95 & 0,95 \\
\hline
\end{tabular}

na krigagem (VIEIRA, 2000). Confirmados os parâmetros do modelo e estimados os números adequados de vizinhos, interpolaram-se valores para os locais onde eles não foram medidos, pelo método da krigagem, usando o programa GEOSTAT (VIEIRA et al., 1983). Os valores estimados por krigagem foram utilizados no programa Surfer (Golden Software, 1999) para confecção dos mapas.

A análise do grau de dependência espacial dos atributos foi realizada segundo CAMBARdella et al. (1994), que considera a dependência espacial fraca quando a relação $\mathrm{C}_{0} /\left(\mathrm{C}_{0}+\mathrm{C}_{1}\right)$ é menor ou igual a 0,25 ; moderada quando está entre 0,25 e 0,75 e forte, quando é maior que 0,75.

Para definir o número de pontos de amostragem necessários para estimar as populações de lagartas e das demais formas biológicas de $D$. saccharalis em cada área estudada, foram considerados dois critérios: o coeficiente de variação e o valor do alcance, obtido na análise geoestatística.

Com o critério do coeficiente de variação $(\mathrm{CV})$, como apresentado por Hoog e Craig (1970), o número de amostras a serem examinadas em cada área foi estimado de maneira que o coeficiente de variação destas $\mathrm{n}$ amostras examinadas fosse de $10 \%, 20 \%, 30 \%$ ou $50 \%$. Para tanto, assumiu-se que o erro-padrão seria igual ao desviopadrão das amostras individuais dividido pela raiz quadrada do tamanho $\mathrm{n}$ a ser determinado (isso é razoável, se amostras forem independentes, ou próximo disso). A fórmula resultante é $\mathrm{n}=(\mathrm{CVi} / \mathrm{CVn})^{2}$, onde $\mathrm{n}$ representa o número de amostras; $\mathrm{CVi}$, o coeficiente de variação observado nos dados originais da área considerada e CVn, o coeficiente de variação que se espera obter $(10 \%, 20 \%$, $30 \%$ ou $50 \%$ ).

Para estimar o número de amostras em função do alcance obtido em cada área, assumiu-se que os pontos de amostragem deveriam ser coletados de maneira que representasse uma área com raio equivalente ao alcance, pois o alcance indica a distância na qual os dados estão correlacionados (Vieira et al., 1983; Souza et al., 1997; VIEIRA, 1997). Nesse caso, não há controle do CV a ser obtido.

\section{RESULTADOS E DISCUSSÃO}

Para condução das análises, as formas biológicas observadas no interior dos colmos foram agrupadas em lagartas (lagartas sadias) e formas biológicas totais (lagartas sadias + pupas + lagartas parasitadas por C. flavipes).

Tanto as populaçóes de lagartas no interior dos colmos como as populaçóes de todas as formas biológicas analisadas tinham coeficientes de variação elevados, com grandes diferenças entre as contagens máxima e mínima (Tabela 2). Segundo Perecin e Barbosa (1994), esse fato é comum em amostragens desse tipo, uma vez que a distribuição esperada, sob a hipótese de dados independentes, é a Poisson (variância = média); a variância será maior que a média, se houver agregação ou contágio.

Valores de assimetria próximos a 0 e de curtose próximos a 3 indicam distribuição normal dos dados. A área 1 tem maiores discrepâncias em relação a esses valores (Tabela 2). Como esperado para esse tipo de contagem, a normalidade dos dados foi rejeitada pelo teste de SHAPIRO e Wilk (1965), mas, de acordo com Cressie (1991), a falta de normalidade dos dados não é impedimento para a realização de análises geoestatísticas; somente é recomendável que não ocorram caudas exageradamente longas. Desta forma, pode-se considerar que os dados apresentam distribuição adequada para análises geoestatísticas.

Os cálculos da relação variância/média, com os valores da tabela 2, sugerem que as agregaçôes são mais fortes na área 2, com sobredispersão maior que $100 \%$ sobre a média; intermediária nas áreas 1 e 4, com sobredispersão ao redor de $50 \%$ sobre a média e ausentes nas três outras áreas. Os mapas (Figuras 2 e 3) parecem concordar com essa afirmação.

Por outro lado, o índice de Morisita foi maior que 1 em todas as áreas estudadas, exceto na área 5, indicando que tanto lagartas sadias como formas biológicas totais distribuem-se de forma agregada ou contagiosa na cultura da cana-de-açúcar, na maioria das situaçóes (Tabela 2).

Entre os 12 semivariogramas calculados (dois em cada área: um para lagartas vivas e outro para formas biológicas 
Tabela 2. Estatísticas descritivas das populaçôes de lagartas e de todos os estádios biológicos (lagartas + pupas + lagartas parasitadas) de $D$. saccharalis nas seis áreas estudadas

\begin{tabular}{|c|c|c|c|c|c|c|c|c|c|c|}
\hline Área & Estádio & $\begin{array}{l}\text { Média } \\
\text { (insetos } \mathbf{m}^{-1} \text { ) }\end{array}$ & $\begin{array}{l}\text { Desvio- } \\
\text { padrão }\end{array}$ & $\begin{array}{l}\text { CV } \\
(\%)\end{array}$ & $\begin{array}{l}\text { Valor mínimo } \\
\text { (insetos } \mathrm{m}^{-1} \text { ) }\end{array}$ & $\begin{array}{l}\text { Valor máximo } \\
\text { (insetos } \mathrm{m}^{-1} \text { ) }\end{array}$ & Simetria & Curtose & $\begin{array}{l}\text { Shapiro- } \\
\text { Wilk test }\end{array}$ & $\begin{array}{l}\text { Índice de } \\
\text { Morisita }\end{array}$ \\
\hline \multirow{2}{*}{1} & lagartas & 0,57 & 0,97 & 170,1 & 0 & 9,0 & 4,86 & 37,84 & $0,57^{* *}$ & $3,01^{* *}$ \\
\hline & todos & 0,58 & 0,99 & 168,4 & 0 & 9,0 & 4,67 & 34,94 & $0,58^{* *}$ & $2,98^{* *}$ \\
\hline \multirow{2}{*}{2} & lagartas & 3,35 & 2,82 & 84,1 & 0 & 17,5 & 2,01 & 6,97 & $0,84^{* *}$ & $1,57^{* *}$ \\
\hline & todos & 3,36 & 2,81 & 83,6 & 0 & 17,5 & 1,99 & 6,92 & $0,82^{* *}$ & $1,55^{*}$ \\
\hline \multirow{2}{*}{3} & lagartas & 0,34 & 0,58 & 172,7 & 0 & 3,0 & 2,44 & 7,04 & $0,63^{* *}$ & $2,50^{* *}$ \\
\hline & todos & 0,39 & 0,61 & 158,1 & 0 & 3,0 & 2,14 & 5,12 & $0,67^{* *}$ & $2,20^{* *}$ \\
\hline \multirow{2}{*}{4} & lagartas & 3,14 & 2,17 & 69,0 & 0 & 11,5 & 0,96 & 1,08 & $0,94^{* *}$ & $1,31^{* *}$ \\
\hline & todos & 3,20 & 2,17 & 67,9 & 0 & 11,5 & 0,96 & 1,08 & $0,94^{* *}$ & $1,30^{* *}$ \\
\hline \multirow{2}{*}{5} & lagartas & 0,24 & 0,36 & 144,4 & 0 & 1,5 & 1,25 & 0,68 & $0,69^{* *}$ & $1,05^{\mathrm{NS}}$ \\
\hline & todos & 0,26 & 0,35 & 135,2 & 0 & 1,5 & 1,13 & 0,46 & $0,71^{* *}$ & $0,92^{\mathrm{NS}}$ \\
\hline \multirow{2}{*}{6} & lagartas & 0,16 & 0,34 & 211,7 & 0 & 2,0 & 2,62 & 8,20 & $0,53^{* *}$ & $2,24^{* *}$ \\
\hline & todos & 0,22 & 0,41 & 193,3 & 0 & 2,0 & 2,35 & 5,82 & $0,58^{* *}$ & $2,44^{* *}$ \\
\hline
\end{tabular}

Significância do teste: NS, ${ }^{*},{ }^{* *}=$ náo significante; $5 \%, 1 \%$.

Tabela 3. Parâmetros para os modelos de semivariogramas esféricos e do jackkinifing, coeficiente de determinação e relaçấo $\mathrm{C}_{0} /\left(\mathrm{C}_{0}+\mathrm{C}_{1}\right)$ para o estádio de lagartas e todos os estádios (lagartas + pupas + lagartas parasitadas ), nas áreas estudadas

\begin{tabular}{|c|c|c|c|c|c|c|c|c|}
\hline \multirow[t]{2}{*}{ Área } & \multirow[t]{2}{*}{ Estádio } & \multicolumn{3}{|c|}{ Parâmetros do semivariograma } & \multicolumn{2}{|c|}{$\begin{array}{l}\text { Parâmetros do Jackknifing } \\
\text { (erros reduzidos) }\end{array}$} & \multirow[t]{2}{*}{$\mathbf{R}^{2}$} & \multirow{2}{*}{$C_{0} /\left(C_{0}+C_{1}\right)$} \\
\hline & & $C_{0}$ & $C_{1}$ & $a(m)$ & média & variância & & \\
\hline \multirow{2}{*}{1} & lagartas & 3,2 & 1,8 & 80 & $-0,0095$ & 0,987 & 0,56 & 0,64 \\
\hline & todos & 3,2 & 1,8 & 70 & $-0,0093$ & 1,008 & 0,62 & 0,64 \\
\hline \multirow{2}{*}{2} & lagartas & 14 & 14,5 & 20 & 0,0118 & 1,028 & 0,62 & 0,49 \\
\hline & todos & 14 & 19 & 22 & 0,0064 & 1,015 & 0,72 & 0,42 \\
\hline \multirow{2}{*}{3} & lagartas & 0,55 & 1 & 23 & 0,0163 & 1,188 & 0,88 & 0,35 \\
\hline & todos & 0,6 & 1 & 20 & 0,0010 & 1,013 & 0,84 & 0,38 \\
\hline \multirow{2}{*}{4} & lagartas & 0 & 19 & 18 & 0,0030 & 1,462 & 0,91 & 0 \\
\hline & todos & 0 & 19 & 18 & 0,0048 & 1,455 & 0,92 & 0 \\
\hline \multirow{2}{*}{5} & lagartas & \multicolumn{7}{|c|}{ efeito pepita puro } \\
\hline & todos & & & & \multicolumn{4}{|c|}{ efeito pepita puro } \\
\hline \multirow{2}{*}{6} & lagartas & & & & \multicolumn{4}{|c|}{ efeito pepita puro } \\
\hline & todos & & & & \multicolumn{4}{|c|}{ efeito pepita puro } \\
\hline
\end{tabular}

totais), aqueles correspondentes às áreas 5 e 6 não se adaptaram a qualquer modelo (efeito pepita puro, Tabela 3), sugerindo que, nestes casos, não há agregação, o que está em concordância com a relação variância/média. Entretanto, de acordo com os índices de Morisita (Tabela 2), somente na área 5 a praga distribuiu-se de forma aleatória. Assim, pode-se inferir que, na área 6, a agregação de D. saccharalis não foi detectada porque está em distâncias muito pequenas, ou seja, as reboleiras eram menores do que as distâncias adotadas entre as amostras. LiebHOLD et al. (1993) citam que a ocorrência de efeito pepita puro em trabalhos com insetos é bastante comum e se deve principalmente ao fato de que a dependência espacial ocorre em uma escala espacial menor do que a escala de amostragem muitas vezes adotada. É preciso considerar ainda que a não-detecção de agregação na área 6 pode ser atribuída também às médias muito baixas. Segundo Perecin e BAR-
BOSA (1994), quando as médias são muito baixas, é difícil separar as distribuições de contágio das aleatórias; segundo estes autores, a distribuição de pragas em campo é um processo bastante dinâmico, sendo comum que as populações iniciem com distribuição aleatória ou mesmo regular (variância menor que a média), continuem aumentado de forma agregada e depois, tomando toda a área, tendem a se tornar novamente aleatória ou regular.

Nas áreas 1, 2 e 4, as populaçôes de D. saccharalis possuem dependência espacial, por qualquer um dos critérios: semivariograma ou relação variância/média.

O modelo esférico foi o que melhor se ajustou aos semivariogramas das áreas 2, 3 e 4, enquanto os dados da área 1 ajustaram-se ao modelo exponencial (Figura 1, Tabela 3). Embora os valores de $\mathrm{R}^{2}$ observados para as áreas $1 \mathrm{e}$ 2 tenham sido menores que 0,75 , os parâmetros estimados para o modelo esférico $\left(\mathrm{C}_{0}, \mathrm{C}_{1}\right.$, a) não foram rejeitados pelo 
(a)

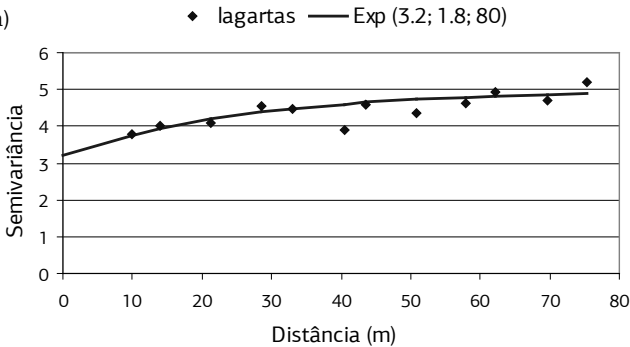

(c)

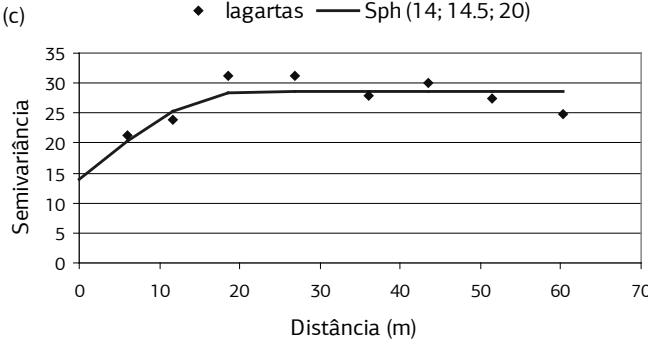

(e)

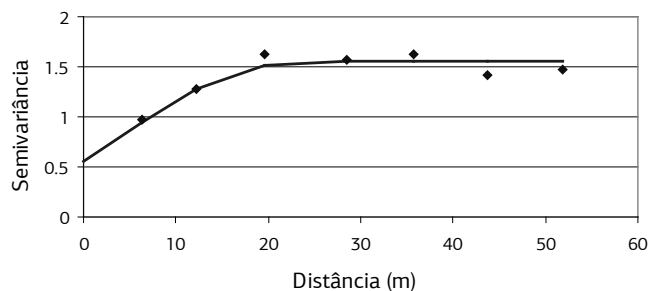

(g)

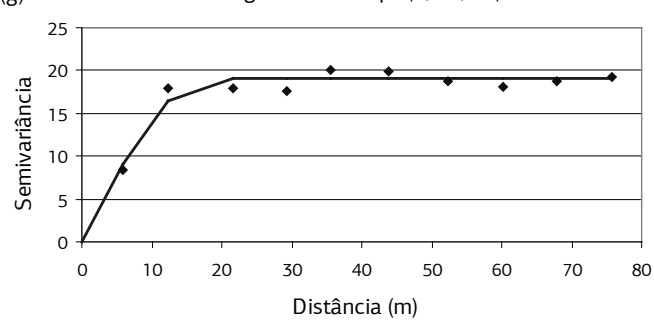

(b)
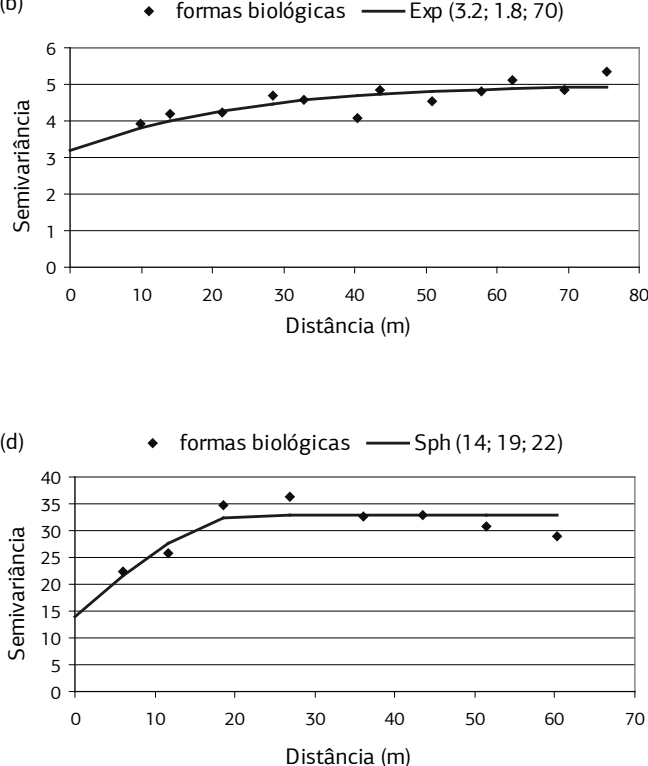

(f)
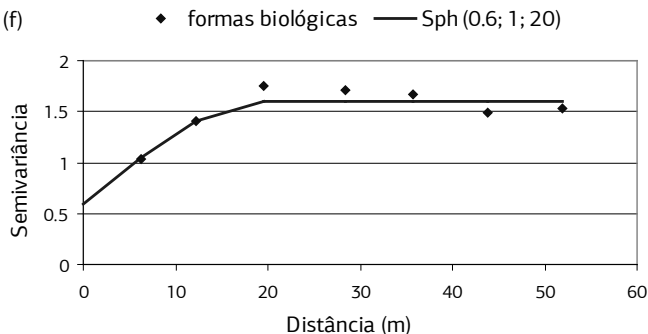

(h) $\quad$ formas biológicas — $\operatorname{Sph}(0 ; 19 ; 18)$

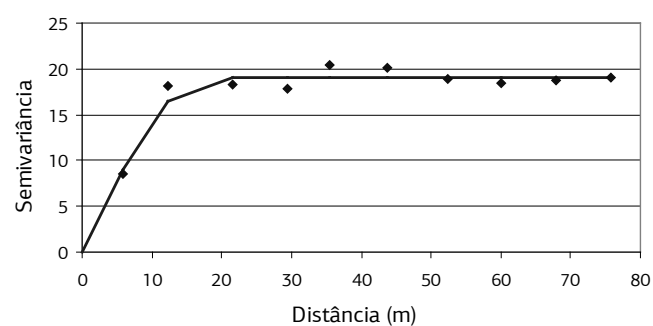

Figura 1. Semivariogramas para populaçóes de lagartas e de formas biológicas totais de $D$. saccharalis, nas áreas 1 (a, b), 2 (c, d), 3 (e, f) e $4(\mathrm{~g}, \mathrm{~h})$. Números entre parênteses são os parâmetros do modelo exponencial ou do esférico $\left(\mathrm{C}_{0}, \mathrm{C}_{1}, \mathrm{a}\right)$.

teste de jackknifing, uma vez que os valores de média dos erros reduzidos estavam próximos de zero e de variância dos erros reduzidos, próximos de 1 (Tabela 3).

O teste de jackknifing também revelou o número ideal de vizinhos a ser usado na krigagem. Consequentemente, a krigagem foi efetuada usando 16 vizinhos, nas áreas 1,3 e 4, e 8 vizinhos, na área 2. Os mapas construídos com base nos dados estáo apresentados nas figuras 2 e 3 .

A porção da variabilidade atribuída à dependência espacial, dada pela relação $\mathrm{C}_{0} /\left(\mathrm{C}_{0}+\mathrm{C}_{1}\right)$, variou de 0 a 0,64 (Tabela 3). Assim, segundo Cambardella et al. (1994), na área 4, os dois parâmetros avaliados proporcionaram forte dependência espacial, enquanto nas áreas 1 a 3, moderada dependência espacial.
Para o estádio de lagartas, o alcance (a), que representa a distância na qual há dependência espacial entre as amostras, variou de $18 \mathrm{~m}$ (área 4) a $80 \mathrm{~m}$ (área 1, Tabela 3), revelando, portanto, que amostras separadas entre si por distâncias menores do que 18 ou $80 \mathrm{~m}$, respectivamente, nas áreas $1 \mathrm{e} 4$, não seriam estatisticamente independentes. Considerando todas as formas biológicas avaliadas, o alcance variou de $18 \mathrm{~m}$ na área $4 \mathrm{a} 70 \mathrm{~m}$ na área 1 .

Uma das principais utilizaçôes dos semivariogramas é na determinação do número ideal de amostras para a estimativa de certo parâmetro, pois, conhecendo-se o alcance da dependência espacial, define-se o raio de amostragem (McBratney e Webster, 1983; Souza et al., 1997; CarvaLHO et al., 2002). Para garantir em uma amostragem futura, 
(a)

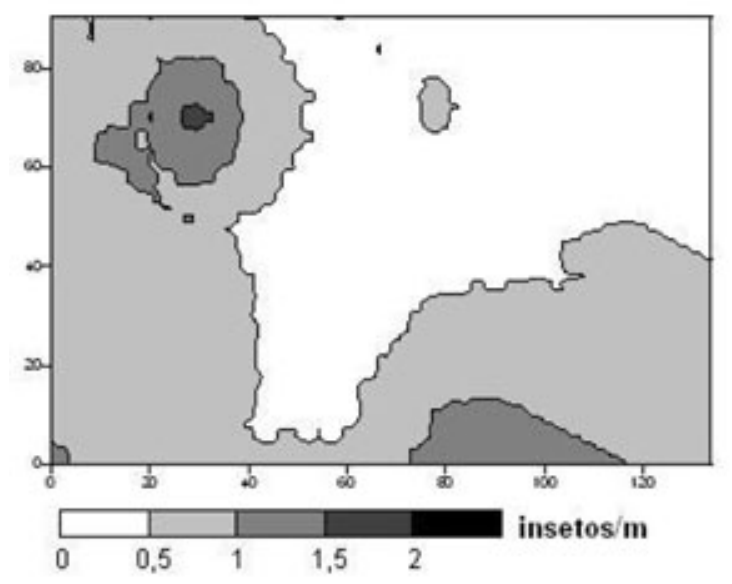

(c)

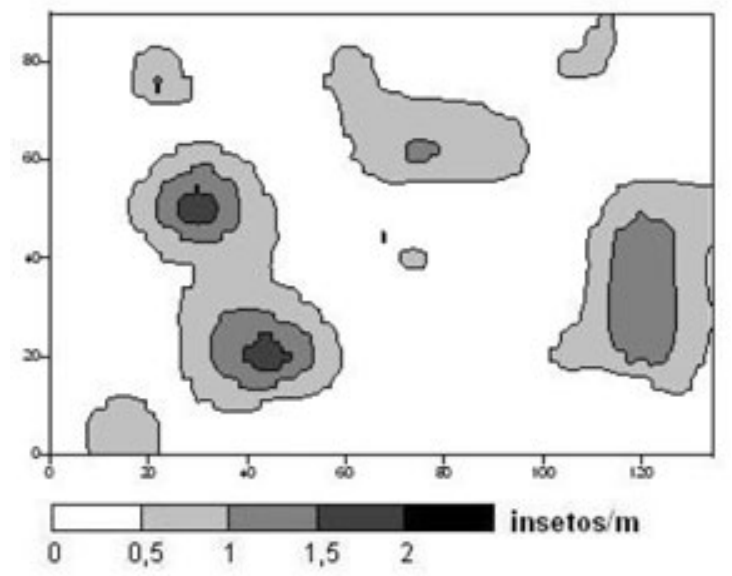

(b)

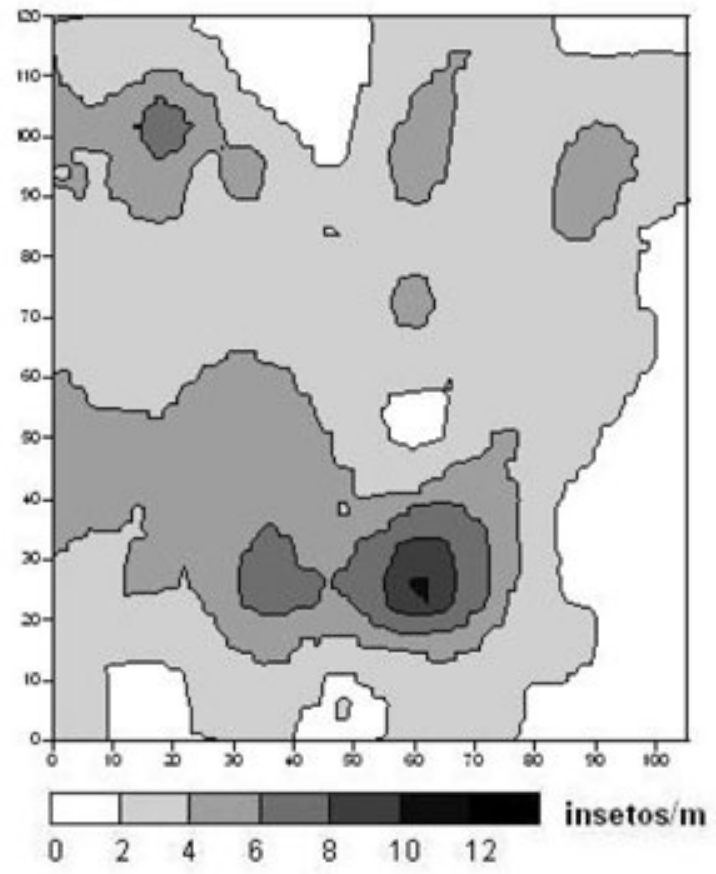

(d)

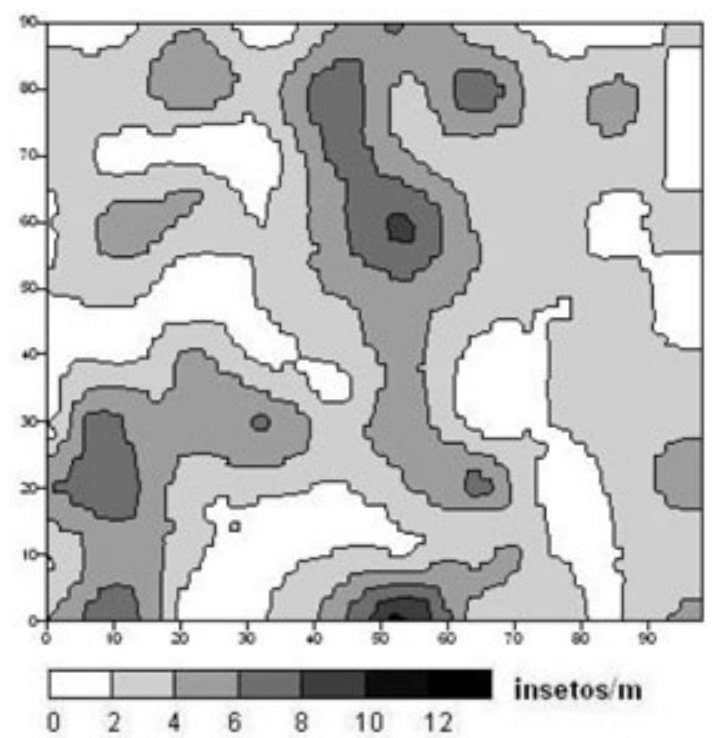

Figura 2. Mapas da distribuição espacial de lagartas nas áreas 1 (a), 2 (b), 3 (c) e 4 (d).

desenvolvida nas mesmas condições da amostragem de referência, a independência dos pontos amostrais, a minimizaçấo do erro-padrão e também para diminuir o número de amostras coletadas, Carvalho et al. (2002) e Souza et al. (2006) sugeriram que o intervalo de amostragem deveria ser duas vezes o valor do alcance. No presente trabalho, assumiu-se que os pontos de amostragem deveriam ser coletados de maneira que representasse uma área com raio equivalente ao alcance. Assim, considerando o alcance, seriam necessários de 0,5 a 9,8 pontos de amostragem por hectare (em média 6 pontos por hectare para representar adequadamente as populaçôes de D. saccharalis nas áreas 1 a 4 (Tabela 4).
A utilização de estudos geoestatísticos para definir planos de amostragem também foi o objetivo de WRIGHT et al. (2002) que trabalharam com Ostrinia nubilalis (Hubner) (Lepidoptera: Crambidae), na cultura do milho e concluíram que, para o estágio larval da praga, as amostras seriam espacialmente dependentes quando coletadas a distâncias de 0,2 a 3,05 m. Com base nesses dados, os autores afirmaram que o esquema de amostragem feito em plantas consecutivas, recomendado até então, não representaria unidades amostrais independentes, violando, portanto, o princípio da casualidade das amostras. 
(a)

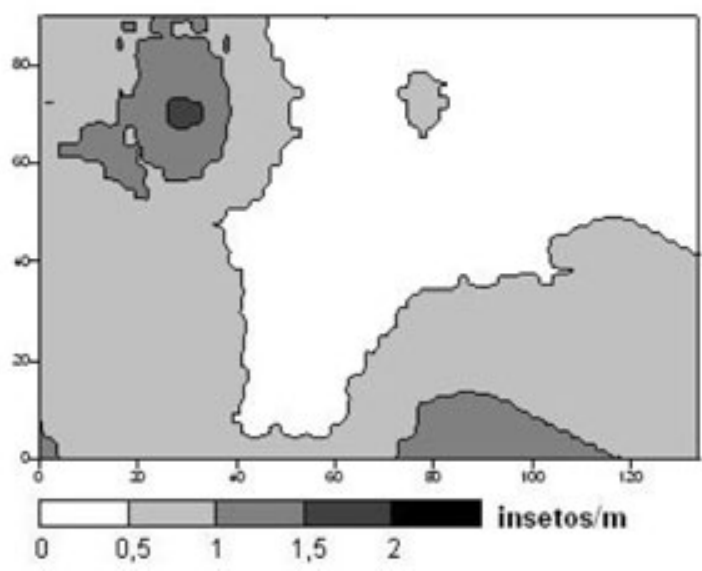

(c)

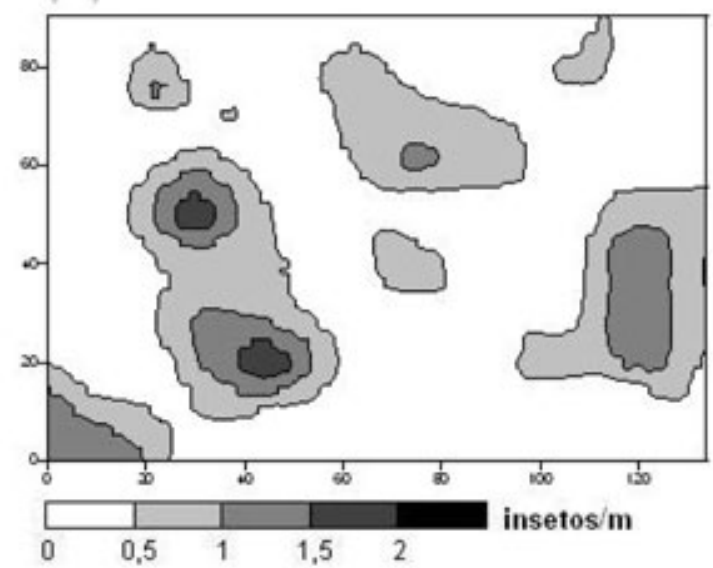

(b)

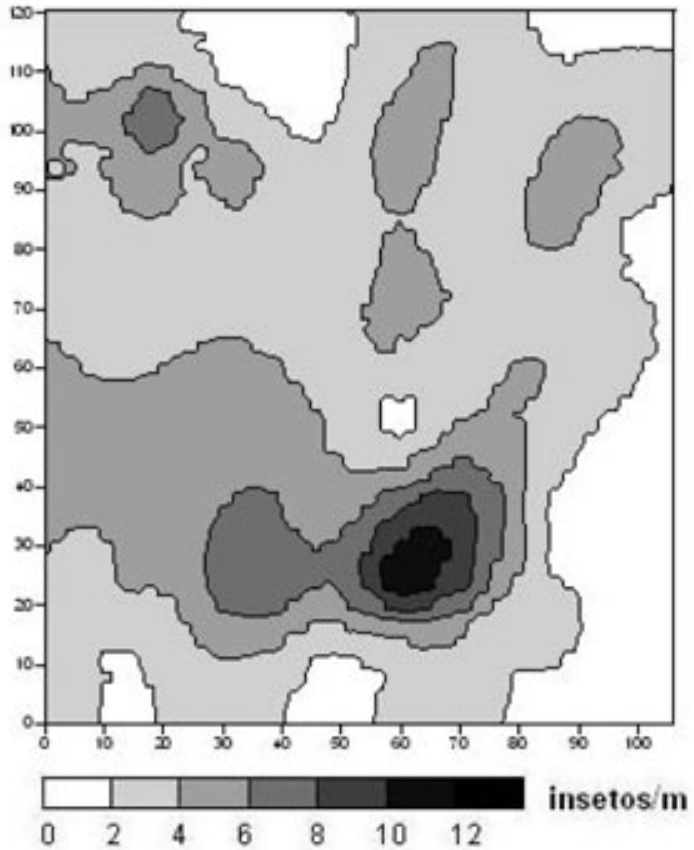

(d)

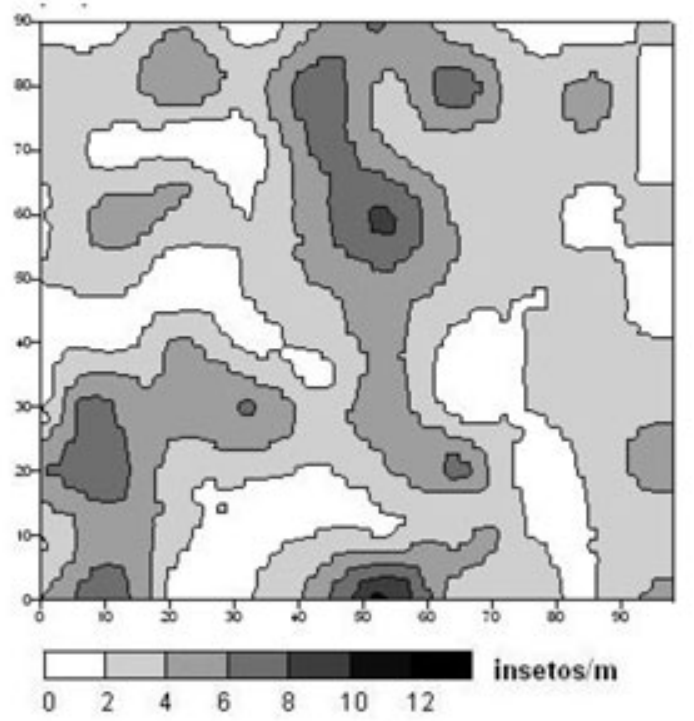

Figura 3. Mapas da distribuição espacial de formas biológicas de $D$. saccharalis nas áreas 1 (a), 2 (b), 3 (c) e 4 (d).

Também com a finalidade de orientar procedimentos de amostragem, FARIAS et al. (2004) realizaram trabalhos em citros e estimaram em 53 a $65 \mathrm{~m}$ o alcance das amostras para estimar as infestaçóes das cigarrinhas Dilobopterus costalimai Young, Acrogonia sp. e Oncometopia facialis Signoret (Hemiptera: Cicadellidae). Esses autores concluíram que pelo menos um ponto de amostragem, representado por uma armadilha, deveria ser feito por hectare, a fim de obter uma estimativa confiável das populações das referidas cigarrinhas.

Trabalhando com Mahanarva fimbriolata Stal (Hemiptera: Cercopidae) em cana-de-açúcar no Brasil, Dinardo-Miranda et al. (2007) constataram alcances variando de 33 a $53 \mathrm{~m}$ e, com base nesse valor, afirmaram que seriam necessários aproximadamente 3 pontos de amostragem por hectare para estimar as populaçóes da praga, a partir de sua segunda geraçáo na área. Os autores, porém, não conseguiram detectar a dependência espacial entre as amostras, em amostragens efetuadas no início do período de ocorrência da praga, quando é necessária a adoção de medidas de controle. Em trabalho posterior, ANjos et al. (2010) sugeriram que seriam necessários 6 pontos de amostragem por hectare para estimar satisfatoriamente as populaçóes de $M$. fimbriolata em cana-de-açúcar, uma vez que o alcance variou de 23 a $55 \mathrm{~m}$ nas áreas amostradas. 
Tabela 4. Número de amostras a serem coletadas por hectare, de acordo com o alcance e com o critério do coeficiente de variação, nas áreas estudadas

\begin{tabular}{|c|c|c|c|c|c|c|}
\hline \multirow{2}{*}{ Área } & \multirow{2}{*}{ Estádio } & \multirow{2}{*}{ Alcance } & \multicolumn{4}{|c|}{ Coeficiente de variação (\%) } \\
\hline & & & 10 & 20 & 30 & 50 \\
\hline \multirow{2}{*}{1} & lagartas & 0,5 & 289 & 72 & 32 & 12 \\
\hline & todos & 0,6 & 282 & 71 & 32 & 11 \\
\hline \multirow{2}{*}{2} & lagartas & 8 & 71 & 18 & 8 & 3 \\
\hline & todos & 7 & 71 & 18 & 8 & 3 \\
\hline \multirow{2}{*}{3} & lagartas & 7 & 295 & 75 & 33 & 12 \\
\hline & todos & 8 & 250 & 63 & 28 & 10 \\
\hline \multirow{2}{*}{4} & lagartas & 10 & 48 & 12 & 5 & 2 \\
\hline & todos & 10 & 46 & 12 & 5 & 2 \\
\hline \multirow{2}{*}{5} & lagartas & - & 207 & 52 & 23 & 8 \\
\hline & todos & - & 182 & 46 & 20 & 7 \\
\hline \multirow{2}{*}{6} & lagartas & - & 445 & 112 & 50 & 18 \\
\hline & todos & - & 372 & 94 & 18 & 15 \\
\hline
\end{tabular}

- Valores não estimados.

No presente trabalho, nas áreas 5 e 6, não foi possível determinar o número ideal de amostras a serem coletadas, utilizando os valores do alcance, porque os semivariogramas apresentaram efeito pepita puro. Nestas situaçôes, o número de amostras a serem tomadas foi estimado somente pelo critério do coeficiente de variação e foi maior quanto menor o coeficiente de variação desejado (Tabela 4).

Para as áreas 1 a 4, nas quais foi possível estimar o número ideal de amostras pelos dois critérios propostos, verifica-se que a utilização do alcance do semivariograma resultou em menor número de amostras a serem coletadas, principalmente ao se comparar com a estimativa feita objetivando obter coeficiente de variação de até 30\% (Tabela 4). Esses resultados corroboram os obtidos por McBratney e Webster (1983), Souza et al. (1997), Carvalho et al. (2002) e Souza et al. (2006), que, embora trabalhando com atributos químicos de solo, afirmaram que o uso do alcance do semivariograma pode reduzir o número de amostras em relação ao uso dos procedimentos amostrais definidos na estatística clássica.

Embora o cultivo da cana-de-açúcar englobe grande variabilidade de ambientes, principalmente relacionados às épocas de plantio e de corte, tratos culturais, variedades cultivadas, entre outros fatores, e embora o trabalho ora apresentado tenha sido desenvolvido em apenas seis dessas inúmeras condiçôes, é imprescindível sugerir um método de amostragem, que contribua para a melhoria da estimativa populacional de formas imaturas de $D$. saccharalis e, consequentemente, de seu manejo. Assim, com base nos dados obtidos, sugere-se a coleta de formas imaturas de $D$. saccharalis em grid de $40 \times 40 \mathrm{~m}$, o que resulta em aproximadamente 6 pontos de amostragem por hectare. Como os pontos de amostragem são representados por $2 \mathrm{~m}$ de sulco, o emprego deste esquema resulta em amostragem de $12 \mathrm{~m}$ de sulco por hectare, o equivalente a $0,18 \%$ dos sulcos do talhão. Este valor é menor do que os $0,28 \%$, que, segundo
TERÁn (1988), representaria satisfatoriamente uma área, quando comparado com a coleta das formas biológicas em área total e com a amostragem de $0,56 \%$ dos sulcos. Porém, embora englobando menor quantidade de metros amostrados, em comparação com o trabalho desenvolvido por Terán (1988), o esquema agora proposto vai de encontro ao que ele sugeriu, por contemplar maior número de pontos de amostragem por hectare. Pelos cálculos mostrados na tabela 4, com esse número de amostras espera-se, na maior parte dos casos, um CV ao redor de $50 \%$ ou menor. Nota-se ainda que, quando o alcance é maior, como ocorre na área 1, o número de amostras sugerido pelo critério do alcance é menor, mas o $\mathrm{CV}$ poderá ser grande, daí a sugestão de conciliar, de alguma forma, os dois critérios, o que pode ser feito utilizando um alcance médio.

\section{CONCLUSÃO}

Em cinco das seis áreas estudadas, as populaçôes de formas imaturas de $D$. saccharalis tiveram distribuição agregada. Com base em um alcance médio dos semivariogramas, sugere-se a coleta de amostras em grid de $40 \times 40 \mathrm{~m}$, o que resulta em aproximadamente 6 pontos de amostragem por hectare, a fim de estimar as populaçóes de formas imaturas da praga em canaviais. Esse número de pontos permitirá, na maioria das áreas, realizar levantamentos com independência e coeficientes de variação menores que 50\%.

\section{REFERÊNCIAS}

ANJOS, I.A.; DINARDO-MIRANDA, L.L.; GARCIA, J.C.; BRAZ, A.J.; TAVARES, C.B.; GEROMEL, R.J.; FERREIRA, S.S.; SOUZA, V.A.; DUARTE, J.C.S. Spatial distribution of sugarcane spittlebug, Mahanarva fimbriolata, in sugarcane 
fields. In: INTERNATIONAL SOCIETY OF SUGAR CANE TECHNOLOGISTS, 1., 2010, Vera Cruz-Mexico. Proceedings... Vera Cruz: ISSCT, 2010. (CD-ROM)

ARRIGONI, E.B. Broca da cana-de-açúcar - Importância econômica e situação atual. In: ARRIGONI, E.B.; DINARDOMIRANDA, L.L.; ROSSETO, R.. Pragas da cana-de-açúcar Importância econômica e enfoques atuais. Piracicaba: STAB/IAC/ CTC, 2002. (CD-ROM)

BOTELHO, P.S.M.; MACEDO, N. Cotesia flavipes para o controle de Diatraea saccharalis. In: PARRA, J.R.P.; BOTELHO, P.S.M.; CORREA-FERREIRA, B.S.; BENTO, J.M.S. (Ed.). Controle biológico no Brasil: Parasitóides e predadores. São Paulo: Manole, 2002. p.409-425.

CAMBARDELLA, C.A.; MOORMAN, T.B.; NOVAK, J.M.; PARKIN, T.B.; KARLEN, D.L.; TURCO, R.F.; KONOPKA, A.E. Field-scale variability of soil properties in central Iowa soils. Soil Science Society of America Journal, v.58, p.1501-1511, 1994.

CARVALHO, J.R.P.; SILVEIRA, P.M.; VIEIRA, S.R. geoestatística na determinação da variabilidade espacial de características químicas do solo sob diferentes preparos. Pesquisa Agropecuária Brasileira, v.37, p.1151-1159, 2002.

CRESSIE, N. Statistics for spatial data. New York: John Wiley, 1991. 900p.

DINARDO-MIRANDA, L.L. Pragas. In: DINARDOMIRANDA, L.L.; VASCONCELOS, A.C.M.; LANDELL, M.G.A. (Ed.). Cana-de-açúcar. Campinas: Instituto Agronômico, 2008. p.349-404.

DINARDO-MIRANDA, L.L.; VASCONCELOS, A.C.M.; VIEIRA, S.R.; FRACASSO, J.V.; GREGO, C.R. Uso da geoestatística na avaliação da distribuição espacial de Mahanarva fimbriolata em cana-de-açúcar. Bragantia, v.66, p.449-455, 2007.

GILES, K.L.; ROYER, T.A.; ELLIOT, N.C.; KINDLER, S.D. Development and validation of a binomial sequential sampling plan for the greenbug (Homoptera: Aplidae) infesting winter wheat in the southern plains. Journal of Economic Entomology, v. 93, p.1522-1530, 2000.

GOLDEN SOFTWARE INC. Surfer for windows. Surfer. Surfer 7.0. Contouring and 3D surface mapping for scientist's engineers. User's Guide. New York: Golden Software, Golden, CO, 1999. 619p.

HOGG, R.V.; CRAIG, A.T. Introduction to mathematical statistics. 3.ed., New York: Macmillan, 1970. 415p.

LIEBHOLD, A.M.; ROSSI, R.E.; KEMP, W.P. Geostatistic and geographic information system in applied insect ecology. Annual Review of Entomology, v.38, p.303-327, 1993

MACBRATNEY, A.B.; WEBSTER, R. How many obsservations are nedeed for regional estimation of soil properties? Soil Science, v.135, p.177-183, 1983.

MACEDO, N.; BOTELHO, P.S.M. Controle integrado da broca da cana-de-açúcar, Diatraea saccharalis (Fabr., 1794) (Lepidoptera, Pyralidae). Brasil Açucareiro, v.106, p. 2-14, 1988.
PERECIN, D.; BARBOSA, J.C. Afinidade entre distribuiçóes de contágio e Poisson para fins práticos de amostragem. Revista de Matemática e Estatística, v.12, p.107-112, 1994.

PRECETTI, A.A.C.M.; TERÁN, F.O.; SÁNCHEZ, A.G. Alteraçôes nas características tecnológicas de algumas variedades de cana-de-açúcar, devidas ao dano da broca Diatraea saccharalis. Boletim Técnico Copersucar, v.41, p.3-8, 1988.

SHAPIRO, S.S.; WILK, M.B. An analysis of variance test for normality: complete samples. Biometrika, v.52, p.591-611, 1965.

SILVEIRA NETO, S.; CARVALHO, R.P.L.; PARANHOS, S.B. Manual de ecologia dos insetos. São Paulo: Ceres, 1976. 419p.

SOUTHWOOD, T.R.E. Ecological methods. 2.ed. New York: John Wiley, 1978. 525p.

SOUZA, L.S.; COGO, N.P.; VIEIRA, S.R. Variabilidade de propriedades físicas e químicas do solo em um pomar cítrico. Revista Brasileira de Ciência do Solo, v.21, p.1-10, 1997.

SOUZA, Z.M.; MARQUES JUNIOR, J.; PEREIRA, G.T.; MONTANARI, R. Otimização amostral de atributos de latossolos considerando aspectos solo-relevo. Ciência Rural, v.36, p.829836, 2006.

STUPIELLO, J.P.O complexo broca-podridão vermelha. STAB Açúcar, Álcool e Subprodutos, v.24, p.14, 2005.

TAYLOR, L.R. Assessing and interpreting the spatial distribution of insects populations. Annual Review of Entomology, v.29, p.321$357,1984$.

TERÁN, F.O. Estudos sobre amostragens populacionais de formas imaturas de Diatraea saccharalis. Boletim Técnico Copersucar, v.41, p.31-36, 1988.

TERÁN, F.O.; SÁNCHEZ, A.G.; PRECETTI, A.A.C.M. Estudos sobre resistência da cana à broca em telado - Primeiros resultados. Boletim Técnico Copersucar, v.29, p. 2-11. 1985.

VIEIRA, S.R. Uso de geoestatística em estudos de variabilidade espacial de propriedades do solo. In: NOVAIS, R.F. (Ed.). Tópicos em Ciência do Solo. Viçosa: Sociedade Brasileira de Ciência do Solo, 2000. p.1-54.

VIEIRA, S.R. Variabilidade espacial de argila, silte e atributos químicos em uma parcela experimental de um latossolo roxo em Campinas (SP). Bragantia, v.56, p.1-17, 1997.

VIEIRA, S.R.; HATFIELD, J.L.; NIELSEN, D.R.; BIGGAR, J.W. Geostatistical theory and application to variability of some agronomical properties. Hilgardia, v.51, p.1-75, 1983.

WRIGHT, R.J., DEVRIES, T.A.; YOUNG, L.J.; JARVI, K.J.; SEYMOUR, R.C. Geostatistical analysis of the small-scale distribution of european corn borer (Lepidoptera: Crambidae) larvae and damage in whorl stage corn. Environmental Entomology, v.31, p.160-167, 2002. 